\title{
Assessment of Outsourcing Management Efficiency Based on Cloud Models
}

\author{
Guo Jie $^{+}$ \\ School of Mechanics \& Civil Engineering China University of Mining \& Technology Beijing, China
}

\begin{abstract}
In order to solve the subjectivity and ambiguity problem in qualitative and quantitative conversion process with traditional assessment methods, application of the cloud model in IT outsourcing project management efficiency assessment methods. To give a final assessment value through a synthesized cloud model merge four indicators, assessment set is determined using a model based on the Model-driven method, and to conduct a comprehensive assessment. Finally, the example shows the feasibility of the method, it provides a new idea for the assessment method based on cloud model.
\end{abstract}

Keywords: cloud model, outsourcing management, uncertain concept, efficiency assessment, synthesized cloud.

\section{Introduction}

Information systems are often seen as providing a core service to the bank customer, for this reason it seems most likely not to be outsourced. However, in the early 1990s, mainframe-based information systems cannot be fast and flexible response to changing customer needs, Continental Bank made the decision to outsource information systems. After 20 years of development, information systems outsourcing has become very common in bank, especially for small and medium-sized banks [1]. At present, for outsourcing management of research focused on identifying and controlling risks, the method for the assessment of management efficiency study is relatively small. Common methods can be divided into subjective and objective methods.

\section{Natural Language Representation Based on Cloud Models}

\subsection{Cloud definition}

Cloud model is a kind of transform model between qualitative concept and quantity by using linguistic value [2]. Cloud is made up of many cloud drops. Every cloud drop is a point, which is one-to-one mapping from qualitative concept to numeral field spaces, namely is a realization of sample reflected quantity.

Let $U$ be a quantitative value on accurate representation of the domain, $C$ is a qualitative concept on $U$. If the quantitative value $x \in U$, and $x$ is a random realization of the qualitative concept $C, x$ of $C$ membership $\mu(x) \in[0,1]$ is a stable tendency of random numbers

$$
\mu: U \rightarrow[0,1] \quad \forall x \in U \quad x \rightarrow \mu(x)
$$

The distribution of $x$ in the domain $U$ called cloud. Each $x$ is called a cloud drop.

Cloud model has three digital characteristics, i.e. Expected value $E x$, Entropy $E n$ and Hyper-Entropy $\mathrm{He}$. The expected value $E x$ of a membership cloud is the position at the universe of

\footnotetext{
+ Corresponding author. Tel.: +8613810522806.

E-mail address: a guojie_cs@126.com.
} 
discourse, corresponding to the centre of the gravity of the cloud. In other word, the element $E x$ in the universe of discourse fully belongs to the linguistic atom represented by the cloud model.

The step of calculating the three digital characteristics is as follows:

$$
\begin{gathered}
\text { (1) } E x=\bar{X}=\frac{1}{n} \sum_{i=1}^{n} x_{i} \\
\text { (2) } S^{2}=\frac{1}{n-1} \sum_{i=1}^{n}\left(x_{i}-\bar{X}\right)^{2} \\
\text { (3) } E n=\sqrt{\frac{\pi}{2}} \times \frac{1}{n} \sum_{i=1}^{n}\left|x_{i}-E x\right| \\
\text { (4) } H e=\sqrt{S^{2}-E n^{2}}
\end{gathered}
$$

\subsection{Forward cloud generator}

Cloud generator is cloud generation algorithm. Forward Cloud Generator is a forward, direct process, enter the three cloud digital characteristics ( $E x, E n, H e$ ) and the need to generate the number of cloud drop, output coordinates of each cloud drop in the domain and each cloud drop represents degree of certainty in concept. Backward cloud generator is the reverse of the forward cloud generator. Enter comply with a distribution of drops, it outputs the corresponding three digital characteristics ( $\mathrm{Ex}, \mathrm{En}, \mathrm{He}$ ).[3]Algorithm of forward normal cloud is as follow:

(1) Produce a normal random number $E n^{\prime}$, according to expected value $E n$ and variance $H e$;

(2) Produce a normal random number $x$, according to expected value $E x$ and variance $E n^{\prime}$;

(3) $Y_{i}=\exp \left(-\frac{\left(X_{i}-E x\right)^{2}}{2\left(E n_{i}^{\prime}\right)^{2}}\right)$;

(4) Gain a cloud drop $i(X i, Y i)$;

Repeat (1)-(4) until enough drops of cloud produced as you like.

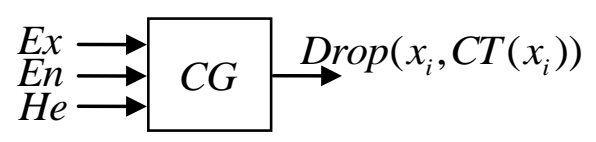

Fig. 1: Forward cloud generator.

\subsection{Normal cloud models}

There are many kinds of cloud models, such as normal cloud. A lot of uncertainty concepts behave normal clouds in social and natural phenomena, so normal cloud has a certain universality [4]. The mathematical expectation curve as follow [5]:

$$
\operatorname{MEC}(x)=\exp \left(-\frac{(x-E x)^{2}}{2 E n^{2}}\right)
$$

Examples of a normal cloud model 1 is obtained by the above method, $\mathrm{Ex}=0, \mathrm{En}=3, \mathrm{He}=0.3, \mathrm{~N}=10000$ is the number of cloud drops. 


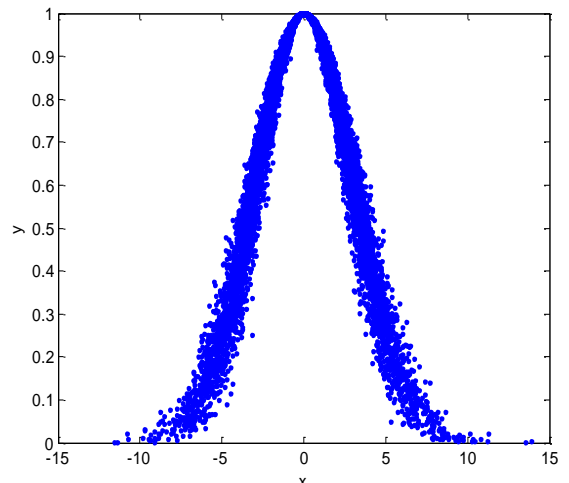

Fig. 2. Normal cloud model.

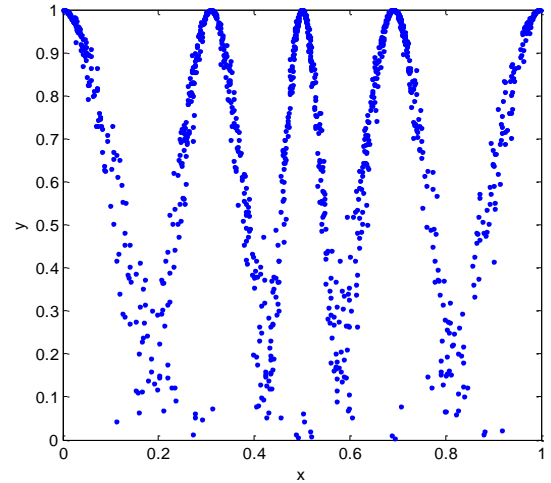

Fig. 3. Assessment cloud.

\section{Research Design}

\subsection{Selecting indicators}

In order to establish IT project outsourcing management assessment standard system, consider three fundamental principles on assessment system: The overall index, do not overlap each other and easy to obtain data, combined with the actual situation case study object, [6], [7] select the item separately from the two sub-indices of input and output indicators, investment management and outsourcing cost components input indicators, the amount of code and project composition quality output indicators. As shown in Table 1.

Table 1. Indicators Table.

\begin{tabular}{|c|l|l|l|}
\hline Target layer & Index code & \multicolumn{1}{c|}{$\begin{array}{c}\text { Index } \\
\text { layer }\end{array}$} & \multicolumn{1}{c|}{ Unit } \\
\hline \multirow{4}{*}{ Assessment Criteria } & I1 & Time-consuming & Man-day \\
\cline { 2 - 4 } & I2 & Outsourcing costs & Ten-thousand yuan \\
\cline { 2 - 4 } & I3 & Lines of code & Rows \\
\cline { 2 - 4 } & I4 & Quality rating & Points \\
\hline
\end{tabular}

It can be seen with different dimensions and different economic significance between the original indicators. If the original index calculated directly composite score, it will be difficult to give a reasonable explanation in economic [8]. Moreover, in order to avoid the correlation between the index caused by the weight bias, a preconditions is between indicators should not have a strong correlation.

\subsection{Assessment method}

Currently, there are two concepts Generation Based on Cloud Model: Data-driven method based on Cloud Transformation and Model-driven method based on golden section method, the former applies to the case of large amount of data. On the division of the domain $[0,1]$, the latter should be used.

Between [0,1], we divided the domain into five assessment levels: "best", "good", "medium", "bad", "worst", Corresponding to the cloud model C1(1, 0.1031, 0.013), C2(0.691, 0.064, 0.008), C3(0.5, 0.039, 0.005), C4(0.309, 0.064, 0.008), C5(0, 0.1031,0.013).[8] As shown in Fig. 3.

\subsection{Synthesized cloud model}

Synthesized cloud can combine two or more flowers of the same type of sub-cloud. Generate a new, more high-level concept of the parent cloud. Its essence is to enhance the concept of the two or more values of the same type of language integrated into a broader concept of linguistic values.

For example, there are Cloud1 $\left(E x_{1}, E n_{1}, H e_{1}\right)$ and Cloud2 $\left(E x_{2}, E n_{2}, H e_{2}\right), C T 1(x)$ and $C T 2(x)$ are expected curve. Get synthesized cloud model. Algorithm is as follows [9], [10]. 


$$
\begin{gathered}
\left\{\begin{array}{l}
E x=\frac{E x_{1} E n_{1}^{\prime}+E x_{2} E n_{2}^{\prime}}{E n_{1}^{\prime}+E n_{2}^{\prime}} \\
E n=E n_{1}^{\prime}+E n_{2}^{\prime} \\
H e=\frac{H e_{1} E n_{1}^{\prime}+H e_{2} E n_{2}^{\prime}}{E n_{1}^{\prime}+E n_{2}^{\prime}}
\end{array}\right. \\
C T_{1}^{\prime}(x)= \begin{cases}C T 1(x) & C T 1 \geq C T 2 \\
0 & \text { Other case }\end{cases} \\
C T_{2}^{\prime}(x)= \begin{cases}C T 2(x) & C T 2 \geq C T 1 \\
0 & \text { Other case }\end{cases} \\
E n_{1}^{\prime}=\frac{1}{\sqrt{2 \pi}} \int C T_{1}^{\prime}(x) d x, E n_{2}^{\prime}=\frac{1}{\sqrt{2 \pi}} \int C T_{2}^{\prime}(x) d x
\end{gathered}
$$

\section{Case Analysis}

\subsection{Computing synthesized cloud process}

To visually illustrate the application process of the assessment method, now give example to be verified. Taking a bank system outsourcing project management, for example, the project manager is bank employee, responsible for project management.

Table 2. Project Management Date

\begin{tabular}{|r|l|l|l|l|l|l|l|l|}
\hline Project No. & \multicolumn{1}{|l}{$\mathbf{I 1}$} & $\mathbf{I 2}$ & $\mathbf{1 3}$ & $\mathbf{I 4}$ & $\mathbf{S 1}$ & $\mathbf{S 2}$ & $\mathbf{R 1}$ & $\mathbf{R 2}$ \\
\hline 1 & 2.7 & 6 & 1933 & 100 & 716 & 16.67 & 0.6692 & 0.6668 \\
\hline 2 & 5.5 & 5 & 3594 & 80 & 653 & 16,12 & 0.6103 & 0.6400 \\
\hline 3 & 2.5 & 6 & 2001 & 60 & 800 & 14.55 & 0.7477 & 0.4800 \\
\hline 4 & 2.5 & 5.5 & 2257 & 80 & 903 & 22.22 & 0.8439 & 0.5820 \\
\hline 5 & 5 & 4.5 & 3126 & 100 & 625 & 18.18 & 0.5841 & 0.8888 \\
\hline 6 & 6.9 & 5.5 & 5794 & 80 & 840 & 22.22 & 0.7850 & 0.7272 \\
\hline 7 & 6.4 & 4.5 & 5009 & 100 & 783 & 13.33 & 0.7318 & 0.8888 \\
\hline 8 & 6.5 & 7 & 6764 & 80 & 1040 & 25 & 0.9720 & 0.5332 \\
\hline 9 & 4.5 & 4 & 4819 & 100 & 1070 & 22.22 & 1.0000 & 1.0000 \\
\hline 10 & 6 & 4.5 & 4960 & 100 & 827 & 14.55 & 0.7729 & 0.8888 \\
\hline 11 & 6.5 & 5.5 & 5292 & 80 & 814 & 20 & 0.7607 & 0.5820 \\
\hline 12 & 6.6 & 5 & 5074 & 100 & 769 & 18.18 & 0.7187 & 0.8000 \\
\hline
\end{tabular}

First, the data were normalized, and obtain the correlation matrix indicators shows that between I1 and I3, I 2 and I4 are two pairs of indicators having a strong correlation.

Table 3. Correlation Matrix.

\begin{tabular}{|l|l|l|l|l|}
\hline & \multicolumn{1}{|c|}{ I1 } & \multicolumn{1}{|c|}{ I2 } & I3 & I4 \\
\hline I1 & 1.0000 & -0.1563 & 0.8979 & 0.2429 \\
\hline I2 & -0.1563 & 1.0000 & 0.0076 & -0.6075 \\
\hline I3 & 0.8979 & 0.0076 & 1.0000 & 0.1559 \\
\hline I4 & 0.2429 & -0.6075 & 0.1559 & 1.0000 \\
\hline
\end{tabular}

Combination of two pairs of indicators having a strong correlation can be described as the unit cost of the rows of code and unit personnel working-time project Quality Score, and standardize new indicators S1 and S2.

Assume the maximum value is the optimal value, after the standardization process to obtain two sub-clouds. R1 and R2 are the result of S1 and S2 apply Linear scaling transformation method. CloudR1 $(0.7664,0.1126,0.0559)$ and CloudR2 $(0.7231,0.1780,0.0592)$ can be calculated. As shown in Figure 4. Three digital characteristics of the synthesized cloud is Cloud $(0.7267,0.2106,0.0589)$. As shown in Fig. 5. 


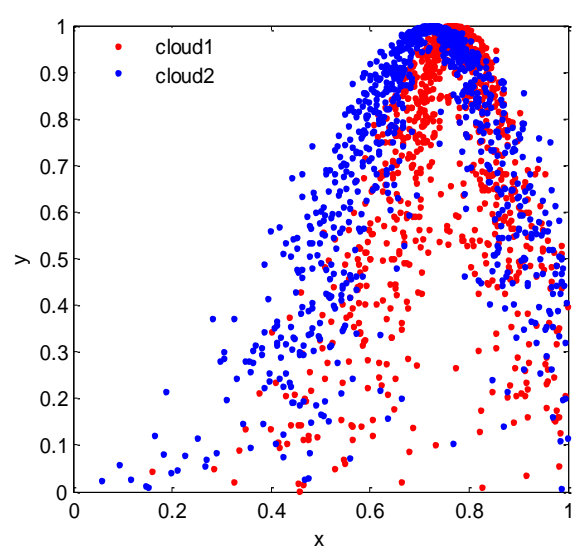

Fig. 4: Sub-cloud.

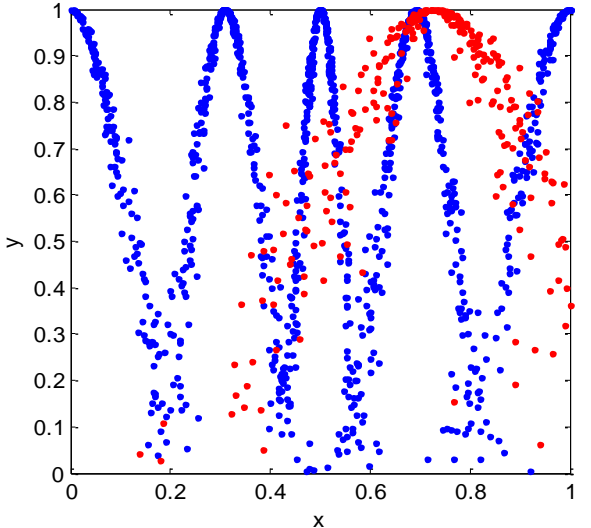

Fig. 5: Standardization cloud.

\subsection{Result analysis}

According to the report statistics, completed a total of 104 projects throughout the year on this system. The distribution of five classes on project assessment is $(18,64,17,5,0)$.

Obviously, this system outsourcing management efficiency slightly better than the second class of "good". The figure shows that the vast majority of the efficiency of outsourcing projects in three classes as "best", "good", "medium". But there are a small parts of projects management efficiency in "bad" class. This result is consistent with the report.

\section{Conclusions}

Through case studies, the availability of this method based on cloud models has been verified. But still there are some problems that need pay attention. This method applies only to the things which obey normal distribution. En usually increases after synthesized cloud computing, which means uncertainty increased.

In this paper, indicator selection is not comprehensive enough, such as it does not reflect the progress of the project to meet the requirements, will further improve the indicator system. Moreover, En and He value would offset when any indicator value is abnormal, results of the accuracy would be impact, de-noise method should be applied.

\section{References}

[1] R.Klepper and W.O.Jones: Outsourcing Information Technology, Systems \& Service. edtied by Prentice Hall (1998)

[2] D.Y.Li, and D.Chang: Mining Association Rules with Linguistic Cloud Models. Journal of Software.Sci.Eng.(2000),11(2):143-158

[3] D.Y.Li, and Y.Du: Artificial Intelligence with Uncertainty. National Defense Industry Press, (2014)

[4] H.Kang, and Y.Fan: Application for Cloud Models in Digital Libraries. Proceedings of the Sixth International Conference on Machine Learning and Cybernetics. Hong Kong: World Academic Union. (2007), pp.3876-3897

[5] F.Zhang and P.Zhang: Assessment of Urban Air Quality Based on Cloud Models. Environmental Science \& Technology. (2009) Vol.32,No.6,pp160-164

[6] L.Ning and M.Li: Evaluation Model for Large and Medium-Sized Industrial Enterprises’ Technological Innovation Capability Based on Factor Analysis Method. Science Research Management.(2011) Vol.32,No.3,pp51-55

[7] S.Li and Y.Gao: Input-output Indicator Selection in the Empirical Study of Bank Efficiency. The Journal of Quantitative \& Technical Economics.(2014)No.4, pp.130-144.

[8] M.Li and G.Chen: Study on Target Standardization Method of Comprehensive Evaluation. Chinese Journal of Management Science.(2004) Vol.12, pp.45-48

[9] D.R.Li, and S.Wang: Spatial Data Mining Theories and Applications. Science Press, (2006)

[10] J.Wang and W.Xiao: An Improved Effectiveness Evaluation Method based on Cloud Model. Fire Control \& Command Control (2010) Vol. 35, No. 7, pp. 139-142. 